\begin{tabular}{lll} 
C O L L O Q U I U M & M A T H E M A T I C U M \\
\hline VoL. 71 & 1996 & No. 1 \\
\hline
\end{tabular}

\title{
CONTINUA WHICH ADMIT NO MEAN
}

BY

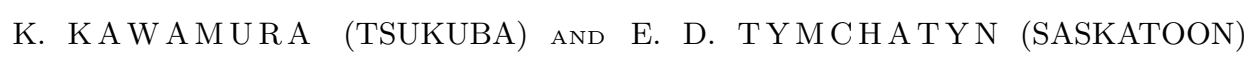

A symmetric, idempotent, continuous binary operation on a space is called a mean. In this paper, we provide a criterion for the non-existence of mean on a certain class of continua which includes tree-like continua. This generalizes a result of Bell and Watson. We also prove that any hereditarily indecomposable circle-like continuum admits no mean.

1. Introduction and preliminaries. A continuum is a compact connected metric space. A continuous map $m: X \times X \rightarrow X$ satisfying the following conditions is called a mean:

(M1) for each $x \in X, m(x, x)=x$,

(M2) $m(x, y)=m(y, x)$ for each $x, y \in X$.

The properties of continua which admit or which do not admit means have been investigated by several authors. The following are some of the known facts.

(1) Every absolute retract admits a mean.

(2) $([\mathrm{S}])$ If a continuum $X$ admits a mean, then $\widetilde{H}^{*}\left(X ; \mathbb{Z}_{2}\right)=0$. In particular, $X$ is unicoherent (see below for the definition), and if it is 1-dimensional, then it is hereditarily unicoherent.

(3) $([\mathrm{Ba}])$ The $\sin (1 / x)$-curve admits no mean, while the dyadic solenoid admits a mean.

(4) $([\mathrm{BeW}])$ There are contractible dendroids and non-contractible dendroids which admit means.

In particular, Bell and Watson [BeW] obtained criteria for the existence and non-existence of means on given continua. A result of the present paper generalizes their non-existence criterion. This generalized criterion does not apply to hereditarily indecomposable continua. Here we also prove that

1991 Mathematics Subject Classification: Primary 54F50; Secondary 54C39.

Key words and phrases: mean, pseudo-arc.

The first named author is supported by an NSERC International Fellowship.

The second named author is supported by the NSERC grant A5616. 
there is no mean on the pseudo-arc nor on any hereditarily indecomposable circle-like continuum. The scheme of both of these proofs is very similar to the one of Bell and Watson.

First, we give some preliminary notions and facts used in the proof.

Definition 1.1. (1) A continuum $X$ is said to be arc-like (circle-like resp.) if, for each $\varepsilon>0$, there is a finite open cover $\mathcal{U}=\left\{U_{1}, \ldots, U_{n}\right\}$ of $X$, called an $\varepsilon$-chain cover ( $\varepsilon$-circular chain cover resp.), with mesh $\mathcal{U}<\varepsilon$ such that $U_{i} \cap U_{j} \neq \emptyset$ if and only if $|i-j| \leq 1(|i-j| \bmod n \leq 1$ resp.). Two points $a$ and $b$ of an arc-like continuum $X$ are called opposite end points if, for each $\varepsilon>0$, an $\varepsilon$-chain cover can be chosen so that $a \in U_{1}$ and $b \in U_{n}$. In this case, $X$ is clearly irreducible between $a$ and $b$ (that is, there is no proper subcontinuum of $X$ containing $a$ and $b$ ). The converse does not hold in general.

(2) A continuum $X$ is said to be unicoherent (indecomposable resp.) if $X=A \cup B$, where $A$ and $B$ are subcontinua of $X$, implies that $A \cap$ $B$ is connected ( $A \subset B$ or $A \supset B$ resp.). If every subcontinuum of $X$ is unicoherent (indecomposable resp.), then $X$ is said to be hereditarily unicoherent (hereditarily indecomposable resp.).

(3) The topologically unique hereditarily indecomposable arc-like continuum is called the pseudo-arc and denoted by $P\left(\left[\mathrm{Bi}_{1}\right]\right)$. Two points $a$ and $b$ of the pseudo-arc $P$ are opposite end points if and only if $P$ is irreducible between $a$ and $b\left(\left[\mathrm{Bi}_{2}\right]\right)$.

Definition 1.2. A continuous map $f: X \rightarrow Y$ is said to be weakly confluent if, for each subcontinuum $B$ of $Y$, there is a subcontinuum $A$ of $X$ such that $f(A)=B$.

TheOREM $1.3([\mathrm{O}])$. Let $Y_{i}$ be arc-like continua $(i=1,2)$. Then for each pair of surjective maps $f_{i}: X_{i} \rightarrow Y_{i}, i=1,2$, the product map $f_{1} \times f_{2}$ : $X_{1} \times X_{2} \rightarrow Y_{1} \times Y_{2}$ is weakly confluent.

TheOREM $1.4([\mathrm{M}])$. Suppose that $f, g:[0,1] \rightarrow[0,1]$ are PL maps of the interval. Then there are PL maps $a, b:[0,1] \rightarrow[0,1]$ such that $f \circ a=g \circ b$. If in addition, $f^{-1}(0)=g^{-1}(0)=\{0\}$ and $f^{-1}(1)=g^{-1}(1)=\{1\}$, then $a$ and $b$ can be chosen so that $a^{-1}(0)=b^{-1}(0)=\{0\}$ and $a^{-1}(1)=b^{-1}(1)=\{1\}$.

LEMma 1.5. Let $X$ be a subcontinuum of an absolute retract $M$. Each mean $m: X \times X \rightarrow X$ on $X$ can be extended to a mean $m^{*}: M \times M \rightarrow M$ on $M$.

Proof. Let $\Sigma(M)=M \times M /(x, y) \sim(y, x)$ be the symmetric product of $M$ and let $\Delta(M)$ be the diagonal set which is naturally contained in $\Sigma(M)$. Notice that $\Sigma(M)$ is metrizable. The mean $m$ defines a retraction of $\Sigma(X)$ onto $\Delta(X)$ which extends to a retraction of $\Sigma(M)$ onto $\Delta(M)$. This defines a mean $m^{*}$ on $M$. 
Definition 1.6. (1) For a subset $A$ of $X \times X, A^{-}$denotes the set $\{(y, x) \mid(x, y) \in A\}$. The subset $A$ is said to be symmetric if $A=A^{-}$.

(2) The $\varepsilon$-neighbourhood of a subset $S$ of a metric space $X$ is denoted by $N(S, \varepsilon)$. The Hausdorff metric induced by a metric $d$ on $X$ is denoted by $d_{\mathrm{H}}$. If $\left\{A_{n}\right\}$ is a sequence of compacta in $X$, then $\operatorname{Lim} A_{n}$ means the limit of $\left\{A_{n}\right\}$ in the Hausdorff metric.

2. Results. Theorem 3.5 of $[\mathrm{BeW}]$ asserts that, if a continuum $X$ contains an $\operatorname{arc} A$ and two sequences of arcs that are "folded in opposite directions with respect to $A$ ", and further, if these sequences converge to $A$ in a "regular way" (essentially, the 0-regularity is assumed), then $X$ admits no mean. Theorem 2.2 below shows that we can remove the hypothesis of the 0-regular convergence. The key tool is the Uniformization Theorem (Theorem 1.4).

Definition 2.1. Let $X$ be a continuum and $A$ be an arc-like subcontinuum of $X$ which has $a$ and $b$ as its opposite end points. A sequence $\left\{A_{n} \mid n \in \mathbb{N}\right\}$ of subcontinua of $X$ is called a folding sequence with respect to the point $a$ if it satisfies the following conditions: for each $n \in \mathbb{N}$, there are two subcontinua $E_{n}$ and $F_{n}$ of $A_{n}$ such that

(1) $A_{n}=E_{n} \cup F_{n}$, and $\operatorname{Lim}\left(E_{n} \cap F_{n}\right)=\{a\}$,

(2) $\operatorname{Lim} E_{n}=\operatorname{Lim} F_{n}=A$.

THEOREM 2.2. Let $X$ be a hereditarily unicoherent continuum which has an arc-like subcontinuum $A$ with the following properties:

(1) $A$ has $a$ and $b$ as its opposite end points, and

(2) there exist folding sequences $\left\{A_{n}\right\}$ and $\left\{B_{n}\right\}$ with respect to $a$ and $b$ respectively.

Then $X$ admits no mean.

The strategy of the proof of the above theorem is the same as that of Theorem 3.5 of [BeW]. We prove the following two lemmas, which are analogues of Lemmas 3.2 and 3.4 of [BeW] respectively.

LeMmA 2.3. Let $m$ be a mean on a continuum $X$ and let $A$ be an arc-like continuum in $X$ which has a and $b$ as opposite end points. Then there exists a subcontinuum $K$ in $A \times A$ intersecting the diagonal $\Delta A$ such that one of the following conditions holds:

(1) $K \cap m^{-1}(a)=\emptyset$ and $K \cap A \times\{a\} \neq \emptyset$, or

(2) $K \cap m^{-1}(b)=\emptyset$ and $K \cap A \times\{b\} \neq \emptyset$.

Proof. Since $a$ and $b$ are opposite end points of $A$, there is an inverse 
limit representation

$$
A=\lim \left(I_{j}, f_{j}: I_{j+1} \rightarrow I_{j}\right)
$$

such that each $I_{j}=\left[0_{j}, 1_{j}\right]$ is the interval with end points $0_{j}$ and $1_{j}$, and

(3) $f_{j}^{-1}\left(0_{j}\right)=\left\{0_{j+1}\right\}, f_{j}^{-1}\left(1_{j}\right)=\left\{1_{j+1}\right\}$, and $f_{j \infty}^{-1}\left(0_{j}\right)=\{a\}, f_{j}^{-1}\left(1_{j}\right)$ $=\{b\}$, where $f_{j \infty}: A \rightarrow I_{j}$ denotes the projection to $I_{j}$.

Let $E_{j}=f_{j \infty} \times f_{j \infty}\left(m^{-1}(a) \cap A \times A\right)$ and $F_{j}=f_{j \infty} \times f_{j \infty}\left(m^{-1}(b) \cap A \times A\right)$ (notice that they are not empty). Taking a subsequence if necessary, we may assume that $E_{j}$ and $F_{j}$ are disjoint. By (3), $E_{j}$ contains $\left(0_{j}, 0_{j}\right)$, and $F_{j}$ contains $\left(1_{j}, 1_{j}\right)$. By Lemma 3.1 of $[\mathrm{BeW}]$, there exists a symmetric subcontinuum $K_{1}$ of $I_{1} \times I_{1}$ intersecting the diagonal $\Delta I_{1}$ such that either

(4.1) $K_{1} \cap I_{1} \times\left\{0_{1}\right\} \neq \emptyset$ and $K_{1} \cap E_{1}=\emptyset$, or

(4.2) $K_{1} \cap I_{1} \times\left\{1_{1}\right\} \neq \emptyset$ and $K_{1} \cap F_{1}=\emptyset$.

Without loss of generality, we assume the first case. Since $f_{1} \times f_{1}$ is weakly confluent by Theorem 1.3, there is a subcontinuum $K_{2}$ of $I_{2} \times I_{2}$ such that $f_{1} \times f_{1}\left(K_{2}\right)=K_{1}$. By the assumption (4.1) and the condition (3), we have $K_{2} \cap I_{2} \times\left\{0_{2}\right\} \neq \emptyset \neq K_{2} \cap\left\{0_{2}\right\} \times I_{2}$ and $K_{2} \cap E_{2}=\emptyset$. Clearly $K_{2}$ intersects the diagonal $\Delta I_{2}$.

Continuing this process, we obtain an inverse sequence

$$
K_{1} \stackrel{f_{1} \times f_{1} \mid K_{2}}{\longleftarrow} K_{2} \stackrel{f_{2} \times f_{2} \mid K_{3}}{\longleftarrow} K_{3} \leftarrow \ldots
$$

whose limit $K$, being naturally identified with a subcontinuum of $A \times A$, satisfies the desired conditions.

LEMMA 2.4. Let $m$ be a mean on a hereditarily unicoherent continuum $X$ and suppose that $A$ is an arc-like subcontinuum of $X$ which has $a$ and $b$ as its opposite end points. If $\left\{A_{n}\right\}$ is a folding sequence with respect to $a$, then for each subcontinuum $K$ of $A \times A$ such that $K \cap \Delta A \neq \emptyset \neq K \cap\{a\} \times A$, we have $K \cap m^{-1}(a) \neq \emptyset$.

Proof. We may assume that $X$ is a subset of the Hilbert cube $I^{\infty}$. The mean $m$ extends to a mean $m^{*}$ on $I^{\infty}$ by Lemma 1.5. Suppose that there is a continuum $K$ in $A \times A$ intersecting $\Delta A$ and $\{a\} \times A$ which is disjoint from $m^{-1}(a)$. By the symmetric property of $m$, we may assume that $K$ is symmetric. Take points $(p, p),(a, q) \in K$ and let $0<4 \varepsilon<d\left(a, m^{*}(K)\right)$. There is a $\delta>0$ such that $\delta<\varepsilon / 4$ and if $d\left((x, y),\left(x^{\prime}, y^{\prime}\right)\right)<\delta$, then $d\left(m^{*}(x, y), m^{*}\left(x^{\prime}, y^{\prime}\right)\right)<\varepsilon / 4$.

Take a map $\varphi: A \rightarrow J=a_{J} b_{J}$ onto an arc $J$ in $I^{\infty}$ which is $\delta / 2$-close to id and such that $\varphi^{-1}\left(a_{J}\right)=\{a\}$ and $\varphi^{-1}\left(b_{J}\right)=\{b\}$. Let $\left\{E_{n}\right\}$ and $\left\{F_{n}\right\}$ be the sequences as in the definition of the folding sequence with respect to $A$ and the point $a$. Take a large $N$ so that $d_{\mathrm{H}}\left(A_{N}, A\right)<\delta / 4$, $d_{\mathrm{H}}\left(E_{N} \cap F_{N}, a\right)<\delta / 4$ and choose sequences $\left\{P_{j}\right\}$ and $\left\{Q_{j}\right\}$ of $\operatorname{arcs}$ in $I^{\infty}$ 
satisfying the following conditions:

(1) $\operatorname{Lim} P_{j}=E_{N}$ and $\operatorname{Lim} Q_{j}=F_{N}$. The arc $P_{j}$ has the end points $s_{j}$ and $t_{j}$, the arc $Q_{j}$ has the end points $s_{j}$ and $u_{j}$.

(2) $P_{j} \cap Q_{j}=\left\{s_{j}\right\}$, where $\lim s_{j} \in E_{N} \cap F_{N}$.

(3) There exists PL maps $h_{j}: P_{j} \rightarrow J$ and $k_{j}: Q_{j} \rightarrow J$ which are $\delta / 2$-close to id.

(4) $h_{j}^{-1}\left(a_{J}\right)=\left\{s_{j}\right\}=k_{j}^{-1}\left(a_{J}\right), h_{j}^{-1}\left(b_{J}\right)=\left\{t_{j}\right\}$, and $k_{j}^{-1}\left(a_{J}\right)=\left\{u_{j}\right\}$.

Applying Theorem 1.4 to $h_{j}$ and $k_{j}$, there exist maps $\alpha_{j}:[0,1] \rightarrow P_{j}$ and $\beta_{j}:[0,1] \rightarrow Q_{j}$ such that

(5) $h_{j} \circ \alpha_{j}=k_{j} \circ \beta_{j}$ and

$\alpha_{j}^{-1}\left(s_{j}\right)=\beta_{j}^{-1}\left(s_{j}\right)=\{0\}$ and $\alpha_{j}^{-1}\left(t_{j}\right)=\beta_{j}^{-1}\left(u_{j}\right)=\{1\}$.

Let $\lambda_{j}=h_{j} \circ \alpha_{j}=k_{j} \circ \beta_{j}$; then by (4), (5) and Theorem 1.3, we have

(6) $\lambda_{j}^{-1}\left(a_{J}\right)=\{0\}$ and $\lambda_{j}^{-1}\left(b_{J}\right)=\{1\}$, and $\lambda_{j} \times \lambda_{j}$ is weakly confluent.

So, there exists a subcontinuum $M_{j} \subset[0,1] \times[0,1]$ such that

$\lambda_{j} \times \lambda_{j}\left(M_{j}\right)=\varphi \times \varphi(K) \supset\left\{(\varphi(p), \varphi(p)),\left(a_{J}, \varphi(q)\right),\left(\varphi(q), a_{J}\right)\right\}$.

By (6), we have $M_{j} \cap([0,1] \times\{0\}) \neq \emptyset \neq M_{j} \cap(\{0\} \times[0,1])$. Therefore $M_{j}$ intersects the diagonal $\Delta[0,1]$. Replacing $M_{j}$ by $M_{j} \cup M_{j}^{-}$, we may assume that $M_{j}$ is symmetric. Take points $\left(x_{j}, x_{j}\right),\left(y_{j}, 0\right),\left(0, y_{j}\right) \in M_{j}$. Define a continuum $V_{j}$ in $\left(P_{j} \cup Q_{j}\right) \times\left(P_{j} \cup Q_{j}\right)$ as follows:

$$
V_{j}=\alpha_{j} \times \alpha_{j}\left(M_{j}\right) \cup \beta_{j} \times \alpha_{j}\left(M_{j}\right) \cup \beta_{j} \times \beta_{j}\left(M_{j}\right) .
$$

To see that $V_{j}$ is indeed a continuum, observe that

$$
\begin{array}{r}
\left(\alpha_{j}\left(x_{j}\right), \alpha_{j}\left(x_{j}\right)\right),\left(\alpha_{j}(0)=s_{j}, \alpha_{j}\left(y_{j}\right)\right) \in \alpha_{j} \times \alpha_{j}\left(M_{j}\right), \\
\left(\beta_{j}(0)=s_{j}, \alpha_{j}\left(y_{j}\right)\right),\left(\beta_{j}\left(y_{j}\right), \alpha_{j}(0)=s_{j}\right) \in \beta_{j} \times \alpha_{j}\left(M_{j}\right), \\
\left(\beta_{j}\left(y_{j}\right), s_{j}=\beta_{j}\left(0_{j}\right)\right),\left(\beta_{j}\left(x_{j}\right), \beta_{j}\left(x_{j}\right)\right) \in \beta_{j} \times \beta_{j}\left(M_{j}\right) .
\end{array}
$$

We may assume that the sequence $\left\{V_{j}\right\}$ converges to a continuum $V$ in $A_{N} \times A_{N}$, and $\alpha_{j}\left(x_{j}\right)$ and $\beta_{j}\left(x_{j}\right)$ converge to $u$ and $v$ respectively, as $j \rightarrow \infty$. By the continuity of $m^{*}$, we have $\operatorname{Lim} m^{*}\left(V_{j}\right)=m(V)$.

By (7), we have $(u, u),(v, v) \in V$ and the condition (M1) implies that $u, v \in m(V)$. Since $X$ is hereditarily unicoherent, $m(V) \cap A_{N}$ is a subcontinuum of $A_{N}$. From the construction, we see that $u \in E_{N}$ and $v \in F_{N}$, hence $m(V) \cap A_{N}$ intersects $E_{N} \cap F_{N}$. Take a point $z \in m(V) \cap E_{N} \cap F_{N}$; then it is $\varepsilon / 4$-close to the point $a$ by the choice of $N$ and $\delta$. A contradiction is derived by proving the following claim.

Claim. $d(m(V), a)>\varepsilon$.

Proof of Claim. First recall that $d\left(m^{*}(K), a\right)>4 \varepsilon$. Since $\varphi$ is $\delta$-close to id, we have $d\left(m^{*}(\varphi \times \varphi(K)), a\right)>4 \varepsilon-\varepsilon / 2>3 \varepsilon$. Take a point 
$(m, n) \in M_{j}$. Since $h_{j} \times h_{j}\left(\alpha_{j}(m), \alpha_{j}(n)\right)=\lambda_{j} \times \lambda_{j}(m, n) \in \varphi \times \varphi(K)$ and $h_{j}$ is $\delta / 2$-close to id, we see that $\alpha_{j} \times \alpha_{j}(m, n) \in N(\varphi \times \varphi(K), \delta)$. By the same argument, we can prove that $V_{j} \subset N(\varphi \times \varphi(K), \delta)$. Hence $\alpha_{j} \times \alpha_{j}\left(M_{j}\right) \subset N(\varphi \times \varphi(K), \delta)$. Therefore, $d\left(m^{*}\left(V_{j}\right), a\right)>3 \varepsilon-\varepsilon=2 \varepsilon$. Taking the limit, we see that $d(m(V), a)>\varepsilon$.

This completes the proof of Lemma 2.4.

Theorem 2.2 follows immediately from the above two lemmas. However, it does not apply to hereditarily indecomposable continua, because it assumes the existence of decomposable subcontinua. The non-existence of means on the pseudo-arc is proved from the following result.

THEOREM 2.5. Let $X$ be a hereditarily unicoherent continuum which contains a pseudo-arc. Then $X$ admits no mean.

COROllary 2.6. The pseudo-arc and each hereditarily indecomposable circle-like continuum admit no mean.

For the proof, we need the following result, which is obtained by the proof of [L], Theorem 1.

THEOREM 2.7. Let $P$ be a pseudo-arc in a metric space $M$ which is irreducible between $x$ and $y$. For each $\varepsilon>0$, there exists $a \delta>0$ such that for each pseudo-arc $Q$ in $M$ which is irreducible between $s$ and $t$ and such that $d_{\mathrm{H}}(P, Q)<\delta, d(x, s)<\delta$ and $d(y, t)<\delta$, there is a homeomorphism $h:(P, x, y) \rightarrow(Q, s, t)$ such that $d(h, \mathrm{id})<\varepsilon$.

For any homogeneous continuum $K$ and for each $\varepsilon>0$, there exists a $\delta>0$, called the Effros number for $\varepsilon$, such that for each pair $x, y$ of points of $K$ with $d(x, y)<\delta$, there exists a homeomorphism $h: K \rightarrow K$ such that $d(h, \mathrm{id})<\varepsilon$ and $h(x)=y$.

Proof of Theorem 2.5. Let $Q_{0}$ be a pseudo-arc in $X$ and let $P \subset Q_{0}$ be a proper sub-pseudo-arc of $Q_{0}$. The strategy of the proof is exactly the same as that of Theorem 2.2. We derive a contradiction by combining Lemma 2.3 with the following assertion:

Assertion. Suppose that the pseudo-arc $P$ above is irreducible between $a$ and $b$ and suppose that $m$ is a mean on $X$. For any subcontinuum $K$ of $P \times P$ with $K \cap \Delta P \neq \emptyset \neq K \cap\{a\} \times P$, we have $K \cap m^{-1}(a) \neq \emptyset$.

Proof of Assertion. Let $K$ be a subcontinuum of $P \times P$ which intersects $\Delta P$ and $\{a\} \times P$, and suppose that $K$ is disjoint from $m^{-1}(a)$. We may assume that $K$ is symmetric.

Let $\varepsilon>0$ be a positive number such that $d(a, m(K))>4 \varepsilon$. Let $\pi_{j}$ : $P \times P \rightarrow P$ be the projection to the $j$ th factor $(j=1,2)$. Observe that $\pi_{1}(K)=\pi_{2}(K)=: R$. Notice that $R$ is also a pseudo-arc. 
First we prove the following:

(1) There exists a continuum $L \subset R \times R$ such that

(1.1) $\pi_{j}(L)=R, j=1,2$,

(1.2) $L \cap \Delta P \neq \emptyset \neq L \cap\{a\} \times P$,

(1.3) $d(m(L), a)>4 \varepsilon$,

(1.4) there is a point $r \in R$ such that $(r, r) \in L$ and $R$ is irreducible between $a$ and $r$.

Indeed, let $(p, p),(a, q) \in K$. Notice that the points $a$ and $p$ need not belong to different composants of $R$. Take a $\beta>0$ such that for any subcontinuum $S$ of $P \times P$ with $d_{\mathrm{H}}(S, K)<\beta$, we have $d(m(S), a)>4 \varepsilon$.

Let $\gamma>0$ be the Effros number for $\beta$ and $R$. There exists a point $r \in R$ which belongs to a different composant of $R$ than the point $a$ and is such that $d(r, p)<\gamma$. By the choice of $\gamma$, there is a homeomorphism $u: R \rightarrow R$ such that $d\left(u, \operatorname{id}_{R}\right)<\beta$ and $u(p)=r$.

Define $L=u \times u(K) \subset R \times R$. Clearly $d_{\mathrm{H}}(L, K)<\beta$, so $d(m(L), a)>4 \varepsilon$. Let $c \in R$ be a point such that $u(c)=a$. Then $(c, d) \in K$ for some $d \in R$, thus $(a, u(d)) \in L$. Therefore $L$ is the desired continuum, and (1) is proved.

In the rest of the proof, we assume that $K=L$ and $p=r$. For convenience, let us summarize the properties of $K$.

(2.1) $\pi_{1}(K)=\pi_{2}(K)=: R \subset P$.

(2.2) $(p, p),(a, q) \in K$ and $d(a, m(K))>4 \varepsilon$.

(2.3) The points $a$ and $p$ belong to different composants in $R$.

Take $\delta$ and $\eta$ with $0<\delta<\varepsilon / 2,0<\eta<\delta$ as follows:

(3.1) If $d\left((x, y),\left(x^{\prime}, y^{\prime}\right)\right)<\delta$, then $d\left(m(x, y), m\left(x^{\prime}, y^{\prime}\right)\right)<\varepsilon / 4$.

(3.2) If a pseudo-arc $T$ in $X$ satisfies $d_{\mathrm{H}}(T, R)<\eta, T$ is irreducible between $s$ and $t$, and $d(a, s)<\eta$ and $d(p, t)<\eta$, then there exists a homeomorphism $h:(R, a, p) \rightarrow(T, s, t)$ such that $d(h$, id $)<\delta / 2$.

(Such an $\eta$ exists by Theorem 2.7.)

We apply Theorem 2.7 (or [L], Theorem 1) to obtain a $\xi>0$ such that for each pseudo-arc $P^{\prime}$ in $X$ with $d_{\mathrm{H}}\left(P, P^{\prime}\right)<\xi$, there is a homeomorphism $g: P \rightarrow P^{\prime}$ such that $d(g$, id $)<\eta / 2$.

Since $P$ is a subcontinuum of $Q_{0}$, there is a pseudo-arc $Q$ in $Q_{0}$ such that $d_{\mathrm{H}}(P, Q)<\xi$. By the choice of $\xi$ above, there exists a homeomorphism $h: P \rightarrow Q$ such that

(4) $d(h$, id $)<\eta / 2$.

Let $c=h(a)$ and $d=h(b)$.

We will define sequences $\left\{Q_{j}^{1}\right\}$ and $\left\{Q_{j}^{2}\right\}$ of subcontinua of $Q$ and sequences of homeomorphisms $\left\{h_{j}^{1}: R \rightarrow Q_{j}^{1}\right\}$ and $\left\{h_{j}^{2}: R \rightarrow Q_{j}^{2}\right\}$ as follows: 
First let $S=h(R)$ and observe that $\{c, h(p)\} \subset S$. By the choice of $h$, $d_{\mathrm{H}}(S, R)<\eta / 2$. Fix two points $p^{1}$ and $p^{2}$ in $S$ such that

(5) $d\left(p^{t}, h(p)\right)<\eta / 2, t=1,2$, and $S$ is irreducible between any pair of points from $\left\{c, p^{1}, p^{2}\right\}$.

For $t=1,2$, let $\left\{Q_{j}^{t}\right\}_{j=1}^{\infty}$ be a sequence of proper subcontinua of $S$ such that

(6) $p^{t} \in Q_{j}^{t}$ for each $j \geq 1$ and $t=1,2$ (in particular, $Q_{i}^{1}$ and $Q_{j}^{2}$ belong to different composants of $S$ for each $i, j)$, and $\operatorname{Lim}_{j} Q_{j}^{t}=S(t=1,2)$.

By taking a subsequence if necessary, we may assume that

(7) $d_{\mathrm{H}}\left(Q_{j}^{t}, R\right)<\eta / 2$ for each $j \geq 1$ and $t=1,2$.

For $t=1,2$, take a sequence $\left\{c_{j}^{t}\right\}_{j=1}^{\infty}$ of points of $Q_{j}^{t}$ such that

(8) $\lim _{j} c_{j}^{t}=c, t=1,2$, and $c_{j}^{t}$ and $p^{t}$ belong to different composants of $Q_{j}^{t}$ for each $j \geq 1$.

Note that, by (4) and (5),

(9) $d\left(p^{t}, p\right)<\eta$,

and we may assume (by (4) and by taking a subsequence if necessary) that

(10) $d\left(c_{j}^{t}, a\right)<\eta$.

By the choice of $\eta$ and (7) and (8), there is a homeomorphism $h_{j}^{t}: R \rightarrow Q_{j}^{t}$ such that

(11) $d\left(h_{j}^{t}, \mathrm{id}\right)<\delta / 2, h_{j}^{t}(a)=c_{j}^{t}, h_{j}^{t}(p)=p^{t}$ for $t=1,2$ and for each $j \geq 1$.

Define a closed set $V_{j} \subset S \times S$ as follows:

$$
V_{j}=h_{j}^{1} \times h_{j}^{1}(K) \cup h_{j}^{1} \times h_{j}^{2}(K) \cup h_{j}^{2} \times h_{j}^{2}(K) .
$$

Recalling that $K$ is symmetric and $(p, p),(a, q),(q, a) \in K$, we have

$$
\begin{aligned}
& \left(p^{1}, p^{1}\right),\left(c_{j}^{1}, h_{j}^{1}(q)\right),\left(h_{j}^{1}(q), c_{j}^{1}\right) \in h_{j}^{1} \times h_{j}^{1}(K), \\
& \left(p^{1}, p^{2}\right),\left(c_{j}^{1}, h_{j}^{2}(q)\right),\left(h_{j}^{1}(q), c_{j}^{2}\right) \in h_{j}^{1} \times h_{j}^{2}(K), \\
& \left(p^{2}, p^{2}\right),\left(c_{j}^{2}, h_{j}^{2}(q)\right),\left(h_{j}^{2}(q), c_{j}^{2}\right) \in h_{j}^{2} \times h_{j}^{2}(K) .
\end{aligned}
$$

We may assume that $\left\{V_{j}\right\}$ converges to a compactum $V$. Although $V_{j}$ is not connected, the conditions (8) and (12) imply that $V$ is a continuum. Since $\left\{p^{1}, p^{2}\right\} \subset m\left(V_{j}\right)$ for each $j \geq 1$, we have $m(V) \supset\left\{p^{1}, p^{2}\right\}$.

Since $X$ is hereditarily unicoherent, $m(V) \cap S$ is a subcontinuum of $S$ intersecting different composants by (6). Thus, $m(V) \cap S=S$, that is, $c \in S \subset m(V)$. Therefore, $d(m(V), a) \leq d(c, a)<\eta<\delta<\varepsilon / 4$.

On the other hand, a computation similar to Lemma 2.4 shows that $d(m(V), a)>\varepsilon$, which contradicts the above.

This completes the proof of the Assertion and hence also of Theorem 2.5. 


\section{REFERENCES}

[Ba] P. Bacon, An acyclic continuum that admits no mean, Fund. Math. 67 (1970), $11-13$.

[BeW] M. Bell and S. Watson, Not all dendroids have means, preprint.

[Bi $\left.{ }_{1}\right] \quad$ R. H. Bing, A homogeneous indecomposable plane continuum, Duke Math. J. 15 (1948), 729-742.

$\left[\mathrm{Bi}_{2}\right] \quad$ - Snake-like continua, ibid. 18 (1951), 653-663.

[C] D. W. Curtis, A hyperspace retraction theorem for a class of half-line compactifications, Topology Proc. 11 (1986), 29-64.

[L] W. Lewis, Observations of the pseudo-arcs, ibid. 9 (1984), 329-337.

[M] J. Mioduszewski, On a quasi-ordering in the class of continuous mappings of a closed interval, Colloq. Math. 9 (1962), 233-240.

[O] L. G. Oversteegen, On products of confluent and weakly confluent mappings related to span, Houston J. Math. 12 (1986), 109-116.

[S] K. Sigmon, Acyclicity of compact means, Michigan Math. J. 16 (1969), 111-115.

Institute of Mathematics University of Tsukuba

Tsukuba-City, Ibaraki 305

Japan
Department of Mathematics and Statistics University of Saskatchewan Saskatoon, Saskatchewan S7N 5E6

Canada

Received 5 April 1995;

revised 24 October 1995 